\title{
Interleukin-6 expression contributes to lapatinib resistance through maintenance of stemness property in HER2-positive breast cancer cells
}

\author{
Wei-Chien Huang ${ }^{1,2,3,4}$, Chao-Ming Hung ${ }^{5,6}$, Ching-Ting Wei ${ }^{5,6, *}$, Tsung-Ming Chen ${ }^{7, *}$, \\ Pei-Hsuan Chien ${ }^{8}$, Hsiao-Lin Pan ${ }^{5}$, Yueh-Ming Lin ${ }^{9}$, Yun-Ju Chen ${ }^{5,8,10}$ \\ ${ }^{1}$ The Ph.D. program for Cancer Biology and Drug Discovery, China Medical University and Academia Sinica, Taichung 404, \\ Taiwan \\ ${ }^{2}$ Graduate Institute of Cancer Biology, China Medical University, Taichung 404, Taiwan \\ ${ }^{3}$ Center for Molecular Medicine, China Medical University and Hospital, Taichung 404, Taiwan \\ ${ }^{4}$ Department of Biotechnology, Asia University, Taichung 413, Taiwan \\ ${ }^{5}$ School of Medicine for International Students, I-Shou University, Kaohsiung 824, Taiwan \\ ${ }^{6}$ Department of General Surgery, E-Da Hospital, Kaohsiung 824, Taiwan \\ ${ }^{7}$ Department and Graduate Institute of Aquaculture, National Kaohsiung Marine University, Kaohsiung 811, Taiwan \\ ${ }^{8}$ Department of Medical Research, E-Da Hospital, Kaohsiung 824, Taiwan \\ ${ }^{9}$ Division of Colorectal Surgery, Department of Surgery, Kaohsiung Chang Gung Memorial Hospital and Chang Gung University \\ College of Medicine, Kaohsiung 833, Taiwan \\ ${ }^{10}$ Department of Biological Science \& Technology, I-Shou University, Kaohsiung 824, Taiwan \\ *These authors have contributed equally to this work
}

Correspondence to: Yun-Ju Chen, email: yjchen0326@isu.edu.tw

Keywords: lapatinib, interleukin-6, HER2, resistance, breast cancer

Received: February 10,2016 Accepted: August 09, $2016 \quad$ Published: August 22, 2016

\section{ABSTRACT}

Lapatinib is an inhibitor of human epidermal growth factor receptor 2 (HER2), which is overexpressed in $\mathbf{2 0 - 2 5 \%}$ of breast cancers. Clinically, lapatinib has shown promising benefits for HER2-positive breast cancer patients; however, patients eventually acquire resistance, limiting its long-term use. In a previous study, we found that interleukin-6 (IL-6) production was increased in acquired lapatinibresistant HER2-positive breast cancer cells. In the present study, we confirmed that lapatinib-resistant cells had elevated IL-6 expression and also maintained both stemness population and property. The increase in IL-6 was required for stemness property maintenance, which was mediated primarily through the activation of signal transducer and activator of transcription 3 (STAT3). Blocking IL-6 activity reduced spheroid formation, cell viability and subsequently overcame lapatinib resistance, whereas stimulation of IL-6 rendered parental cells more resistant to lapatinibinduced cytotoxicity. These results point to a novel mechanism underlying lapatinib resistance and provide a potential strategy to overcome resistance via IL-6 inhibition.

\section{INTRODUCTION}

Breast cancer is the most common cancer in women worldwide and its incidence is increasing yearly. It can be classified into several subtypes according to the expression of several biomarkers. Overexpression of human epidermal growth factor receptor 2 (HER2, also known as Neu, ErbB2, EGFR2) is found in approximately $20-25 \%$ of breast cancer patients $[1,2]$. HER2 is a member of HER/ErbB family and plays a critical role in the cancer progression via its receptor tyrosine kinase (RTK) activity [3]. HER2 is also a positive regulator of the cancer stem cell (CSC) population in HER2-positive breast cancers [4, 5]. HER2 overexpression increases, whereas HER2 inhibition decreases, the breast tumor initiating cell (BTIC) population in vitro [6]. Consistent with this finding, a positive correlation between HER2 expression and CSC frequency is also observed in human breast cancers [7]. These important features render 
HER2-positive breast cancer a highly malignant state with a poor prognosis $[8,9]$. Blockage of HER2 activity with the kinase inhibitor lapatinib (GW572016, Tykerb ${ }^{\circledR}$ ) has shown remarkable clinical efficacy in most patients with HER2-positive breast tumors $[10,11]$, but these patients eventually develop resistance. The mechanism underlying acquired resistance to lapatinib has not yet been completely elucidated [11-13].

The cytokine interleukin-6 (IL-6) was initially identified as a critical regulator of the immune response $[14,15]$. However, it also activates downstream signaling pathways, such as the JAK/STAT pathway, to enhance tumor progression [16]. Elevated expression of IL-6 or its receptor are commonly found in many cancer types, including breast cancer, and are associated with poor prognosis $[17,18]$. Furthermore, IL-6 expression renders tumor cells resistant to anti-cancer therapies [19-21]. Interestingly, Hartman et al., report that HER2 enhances IL6 transcription, resulting in the activation of the IL-6/JAK/STAT3 autocrine loop, which plays a pivotal role in the carcinogenesis of HER2positive breast cancer [22]. Up-regulation of IL-6 enhanced HER2-mediated mammosphere formation [23], as well as the tumorigenic conversion of mammary stem cells $\left(\mathrm{CD} 44^{\mathrm{hi}} \mathrm{CD} 24^{\text {lo }}\right)$, by activating Jagged-1/Notch-3 signaling $[24,25]$. These findings indicate the critical role of IL-6 in BTIC expansion in HER2-positive breast cancer cells.

Our previous study showed that long-term treatment of lapatinib in breast cancer cells with or without HER2 expression enhances $\mathrm{NF}-\kappa \mathrm{B}$ activation and subsequently results in the expression of $\mathrm{NF}-\kappa \mathrm{B}$ downstream genes, including IL6. Furthermore, increased IL-6 production was observed in HER2-positive breast cancer cells with acquired lapatinib resistance [26]. However, it remains unclear whether increased IL-6 expression determines the sensitivity of HER2-positive breast cancer cells to lapatinib. In this study, we confirmed that acquired lapatinib-resistant HER2-positive breast cancer cells had elevated IL-6, and that elevated IL-6 maintained the stemness population and property within these resistant cells through the activation of signal transducer and activator of transcription 3 (STAT3). Blocking IL-6 activity reduced the BTIC population and cell viability of these resistant cells. However, stimulation of IL-6 made parental cells more resistant to lapatinib treatment. Taken together, these results provide evidence that, when the survival signal of breast cancer cells is switched from HER2 to IL-6 signaling, lapatinib resistance may be acquired. Moreover, blockage of IL- 6 activity may be a potential strategy to overcome this resistance.

\section{RESULTS}

\section{Maintenance of stemness population and property is observed within acquired lapatinib-resistant clones}

Our previous results indicated that long-term treatment of lapatinib enhances NF- $\kappa \mathrm{B}$-dependent gene expression, including the expression of IL6 [26]. Here, we confirmed the elevation of IL6 expression in HER2-positive breast cancer cells with acquired resistance to long-term lapatinib treatment. We found increased expression of IL6 mRNA and IL-6 protein in two acquired lapatinib-resistant clones, SkBr3/Lap\#6 and SkBr3/Lap\#9 (Figures 1A and 1B). Since IL-6 plays a critical role in BTIC expansion in HER2-positive breast cancer cells, we next examined the stemness property of these resistant cells using spheroid formation and aldehyde dehydrogenase (ALDH) activity assays. As shown in Figure 1C, lapatinib treatment reduced spheroid formation in parental $\mathrm{SkBr} 3$ cells, whereas the ability to form spheroids was restored in both lapatinibresistant SkBr3/Lap\#6 (left panel) and SkBr3/Lap\#9 (right panel) cells. Consistent with this observation, ALDH activity was also decreased in lapatinib-treated $\mathrm{SkBr} 3$ cells, but was maintained in SkBr3/Lap\#6 cells (Figure 1D). The expression of stemness markers, CD133 and Nanog was also increased in SkBr3/Lap\#6 cells (Figure $1 \mathrm{E})$. Furthermore, the population of cancer stem cell marker CD $44^{\text {high }} / \mathrm{CD} 24^{\text {low }}$ expression was slightly elevated in $\mathrm{SkBr3} / \mathrm{Lap} \# 6$ cells as compared with that in $\mathrm{SkBr} 3$ cells (Figure 1F). These results suggest that IL-6 expression stemness property and population were maintained in acquired lapatinib-resistant cells.

\section{IL-6 is required for the maintenance of stemness property in acquired lapatinib-resistant cells}

We next investigated whether IL-6 is required for the maintenance of stemness property in acquired lapatinibresistant cells using an IL6 siRNA and a neutralizing IL-6 antibody. As shown in Figure 2A, spheroid formation in $\mathrm{SkBr} 3 / \mathrm{Lap \# 6}$ and $\mathrm{SkBr3} / \mathrm{Lap} \# 9$ cells was attenuated (upper panel) when IL6 expression was depleted by siRNA (lower panel). In addition, ALDH activity was also decreased in SkBr3/Lap\#6 cells when IL6 expression was blocked by siRNA (Figure 2B). Using an IL-6 neutralizing antibody to block IL-6 activity, there was attenuation in both spheroid formation (Figure 2C, upper panel) and ALDH activity (Figure 2C, middle panel) in $\mathrm{SkBr3/Lap \# 6} \mathrm{cells.}$ IL-6 activity was evaluated by examining its downstream signaling molecule, STAT3 tyrosine 705 phosphorylation (Figure 2C, lower panel). In contrast to these results, both spheroid formation (Figure 2D, upper panel) and ALDH activity (Figure 2D, middle panel) of parental $\mathrm{SkBr} 3$ cells were increased when cells were stimulated with recombinant IL-6 (Figure 2D, lower panel). Furthermore, the results from in vitro transwell assays showed that both migration and invasion of $\mathrm{SkBr} 3 / \mathrm{Lap} \# 6$ cells were blocked when IL6 expression was silenced (Figure 2E). Similar results were also observed in $\mathrm{SkBr} 3 / \mathrm{Lap} \# 9$ cells (Figure 2F). The analysis of marker expression of epithelial-to-mesenchymal transition also revealed consistent results (Figure $2 \mathrm{G}$ ). These results indicate that IL-6 activity is required for the maintenance of stemness property as well as migration/invasion in acquired lapatinib-resistant cells. 


\section{STAT3 is the major downstream effector for IL- 6-mediated maintenance of stemness property}

IL-6 regulates biological responses by activating several intracellular signaling effectors, such as STAT3, ERK, and Akt. We further explored the molecular mechanism underlying IL-6-mediated effects in these lapatinib-resistant cells by examining the effects of silencing IL6 on these downstream signaling effectors. Only tyrosine 705 phosphorylation of STAT3 was attenuated when $\mathrm{SkBr} 3 / \mathrm{Lap} \# 6$ cells lost IL6 expression (Figure 3A, left panel). Similar results were also observed in $\mathrm{SkBr} 3 / \mathrm{Lap} \# 9$ cells (Figure 3A, right panel). Importantly, the tyrosine 705 phosphorylation of STAT3 in resistant cells was no longer altered by lapatinib treatment (Figure 3B). Next, we investigated whether STAT3 activation is required for IL-6-mediated regulation in these resistant cells. As shown in Figure 3C, when STAT3 expression was depleted by siRNA (lower panel), spheroid formation was
A

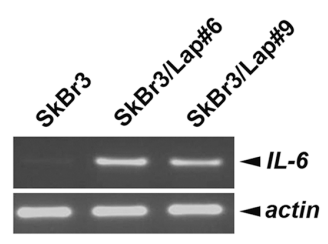

C

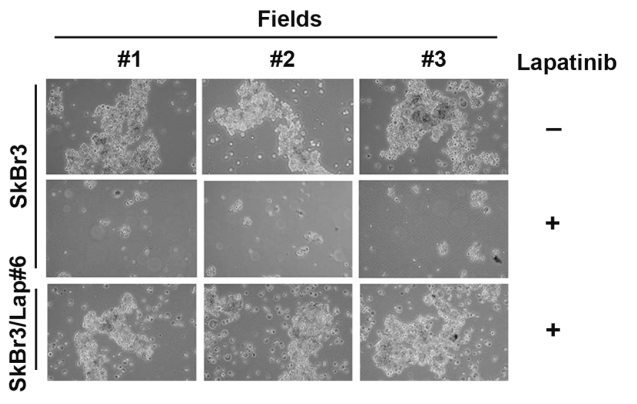

D

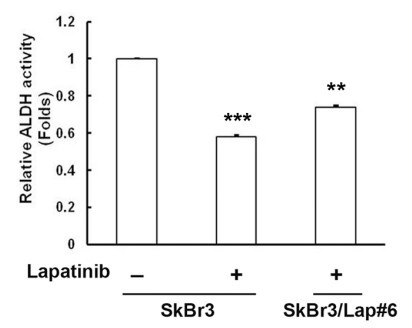

B
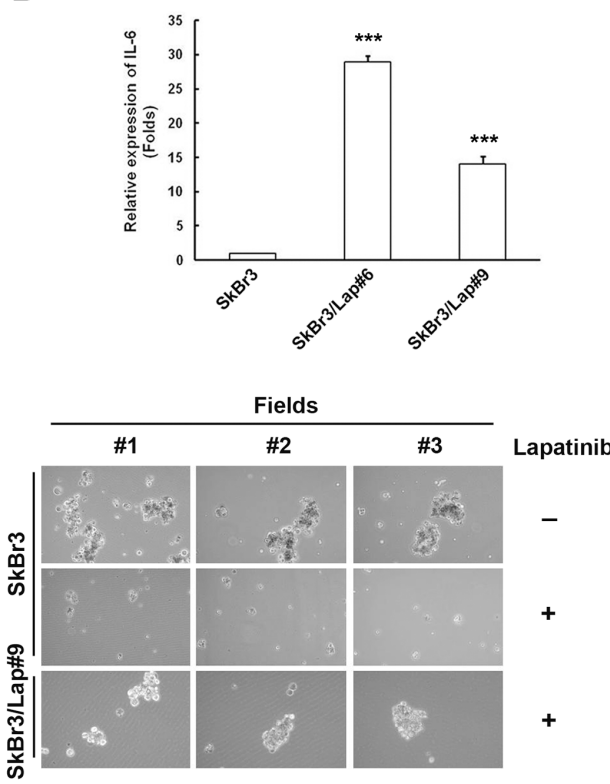

E

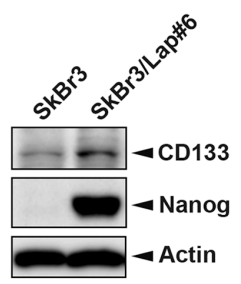

$\mathbf{F}$

\begin{tabular}{|l|c|c|c|c|} 
& CD44 $^{\text {low }} / C D 24^{\text {low }}$ & CD44 $^{\text {high }} / C^{2} 4^{\text {low }}$ & CD44 $^{\text {low }} /$ CD24 $^{\text {high }}$ & CD44 $^{\text {high }} /$ CD24 $^{\text {high }}$ \\
\hline SkBr3 & $18.19 \%$ & $1.21 \%$ & $64.43 \%$ & $16.17 \%$ \\
\hline SkBr3/Lap\#6 & $76.11 \%$ & $3.46 \%$ & $17.75 \%$ & $2.68 \%$ \\
\hline
\end{tabular}

Figure 1: Maintenance of stemness population and property is observed within acquired lapatinib-resistant clones. A. Total RNA was extracted from SkBr3, SkBr3/Lap\#6 and SkBr3/Lap\#9 cells and subjected to RT-PCR. mRNA expression of IL6 and internal control ACTIN was examined by $1 \%$ electrophoresis. B. Media from SkBr3, SkBr3/Lap\#6 and SkBr3/Lap\#9 cells were collected to examine IL- 6 production by ELISA. Statistical analysis was performed by Student's t test. ***, $\mathrm{p}<0.001$ as compared to control group. C. $\mathrm{SkBr} 3, \mathrm{SkBr} 3 / \mathrm{Lap} \# 6$ and $\mathrm{SkBr} 3 / \mathrm{Lap} \# 9$ cells were cultured at a density of $2 \times 10^{4}$ cells $/ \mathrm{ml}$ in ultra-low attachment plates with spheroidinducing medium. Cells were treated with $1 \mu \mathrm{M}$ lapatinib for 7 days, and spheroid formation observed under microscope. D. SkBr3 and $\mathrm{SkBr} 3 / \mathrm{Lap} \# 6$ cells were treated with $1 \mu \mathrm{M}$ lapatinib for 1 day, then re-seeded and $2 \times 10^{6}$ cells per group were subjected to an ALDH activity assay. Statistical analysis was performed by Student's t test. **, $\mathrm{p}<0.01 ; * * *, \mathrm{p}<0.001$ as compared to control group. E. The whole cell lysates of both $\mathrm{SkBr} 3$ and $\mathrm{SkBr} 3 / \mathrm{Lap} \# 6$ cells were harvested and subjected to western blot analysis with indicated antibodies. F. SkBr3 and $\mathrm{SkBr} 3 / \mathrm{Lap} \# 6$ cells were fixed and subjected to flow cytometry with indicated antibodies. 
A
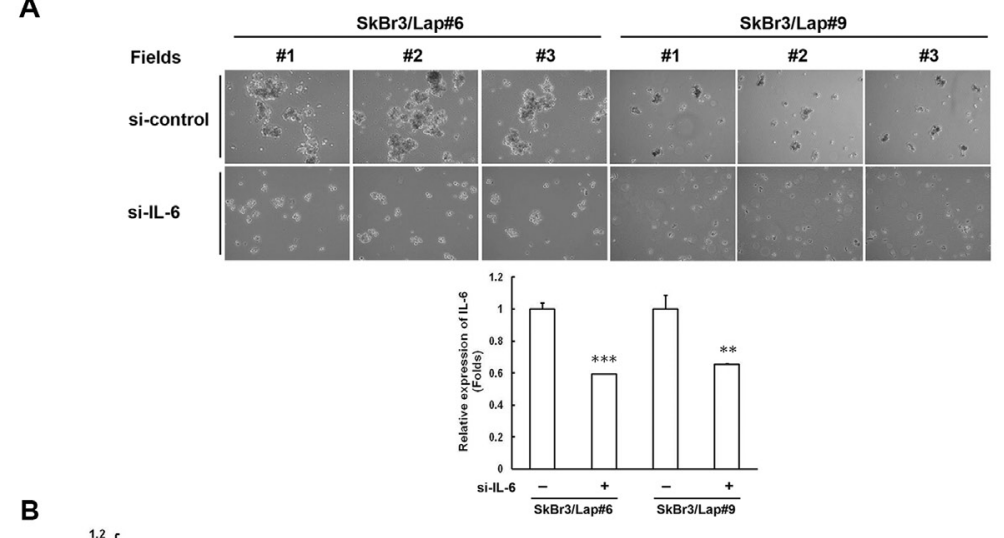

B

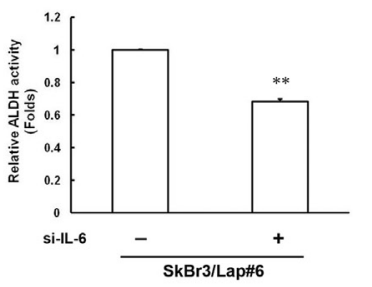

C
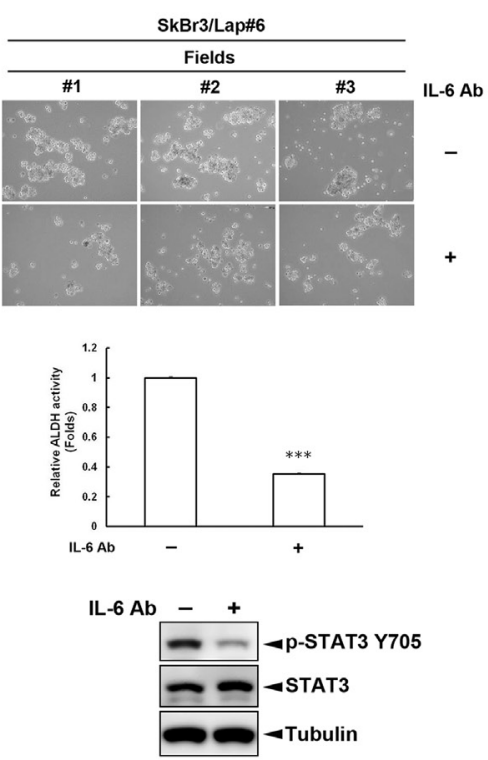

D
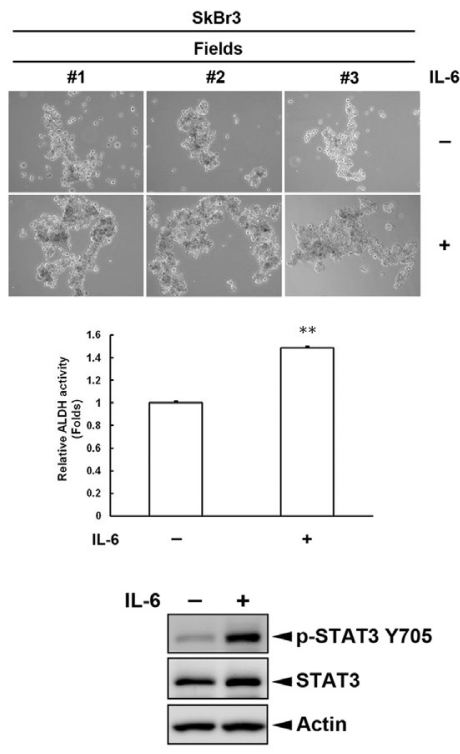

Figure 2: IL-6 is required for the maintenance of stemness property in cells with acquired lapatinib-resistance. A. $\mathrm{SkBr3} / \mathrm{Lap} \# 6$ and $\mathrm{SkBr} 3 / \mathrm{Lap} \# 9$ cells were transfected with si-control or si-IL6. Two days later, cells were trypsinized and $4 \times 10^{4}$ cells were cultured in ultra-low attachment plates with spheroid-inducing medium, followed by observation of spheroid formation under a microscope. Gene silencing of $I L 6$ was measured by ELISA. Statistical analysis was performed by Student's t test. **, $\mathrm{p}<0.01 ;{ }^{* * *}, \mathrm{p}<0.001$ as compared to each control group. B. SkBr3/Lap\#6 cells were transfected with si-control or si-IL6. Two days later, cells were trypsinized and $4 \times 10^{4}$ cells were cultured in ultra-low attachment plates with spheroid-inducing medium for 7 days. Spheroids were collected and 2 $\mathrm{x} 10^{6}$ spheroids were subjected to an ALDH activity assay. Statistical analysis was performed by Student's t test. **, $\mathrm{p}<0.01$ as compared to control group. C. SkBr3/Lap\#6 cells at a density of $2 \times 10^{4}$ cells $/ \mathrm{ml}$ were cultured in ultra-low attachment plates with spheroid-inducing medium and treated with $10 \mu \mathrm{g} / \mathrm{ml} \mathrm{IL}-6$ antibody for 7 days, followed by observation of spheroid formation under a microscope. Then, 2 $\mathrm{x} 10^{6}$ spheroids were collected and subjected to an ALDH activity assay. Cell lysates from SkBr3/Lap\#6 cells treated with $10 \mu \mathrm{g} / \mathrm{ml} \mathrm{IL-6}$ antibody were harvested and western blots used to examine phosphorylation of STAT3 using indicated antibodies. Statistical analysis was performed by Student's t test. ${ }^{* * *}, \mathrm{p}<0.001$ as compared to control group. D. SkBr3 cells at a density of $2 \times 10^{4}$ cells $/ \mathrm{ml}$ were cultured in ultra-low attachment plates with spheroid-inducing medium and treated with $20 \mathrm{ng} / \mathrm{ml} \mathrm{recombinant} \mathrm{IL-6} \mathrm{protein} \mathrm{for} 7$ days, followed by the observation of spheroid formation under microscope. Then, $2 \times 10^{6}$ spheroids were collected and subjected to an ALDH activity assay. Cell lysates from $\mathrm{SkBr} 3$ cells treated with $20 \mathrm{ng} / \mathrm{ml}$ recombinant IL-6 protein were harvested and western blots used to examine phosphorylation of STAT3 using indicated antibodies. Statistical analysis was performed by Student's $t$ test. ${ }^{* *}, \mathrm{p}<0.01$ as compared to control group.

(Continued) 
$\mathbf{E}$

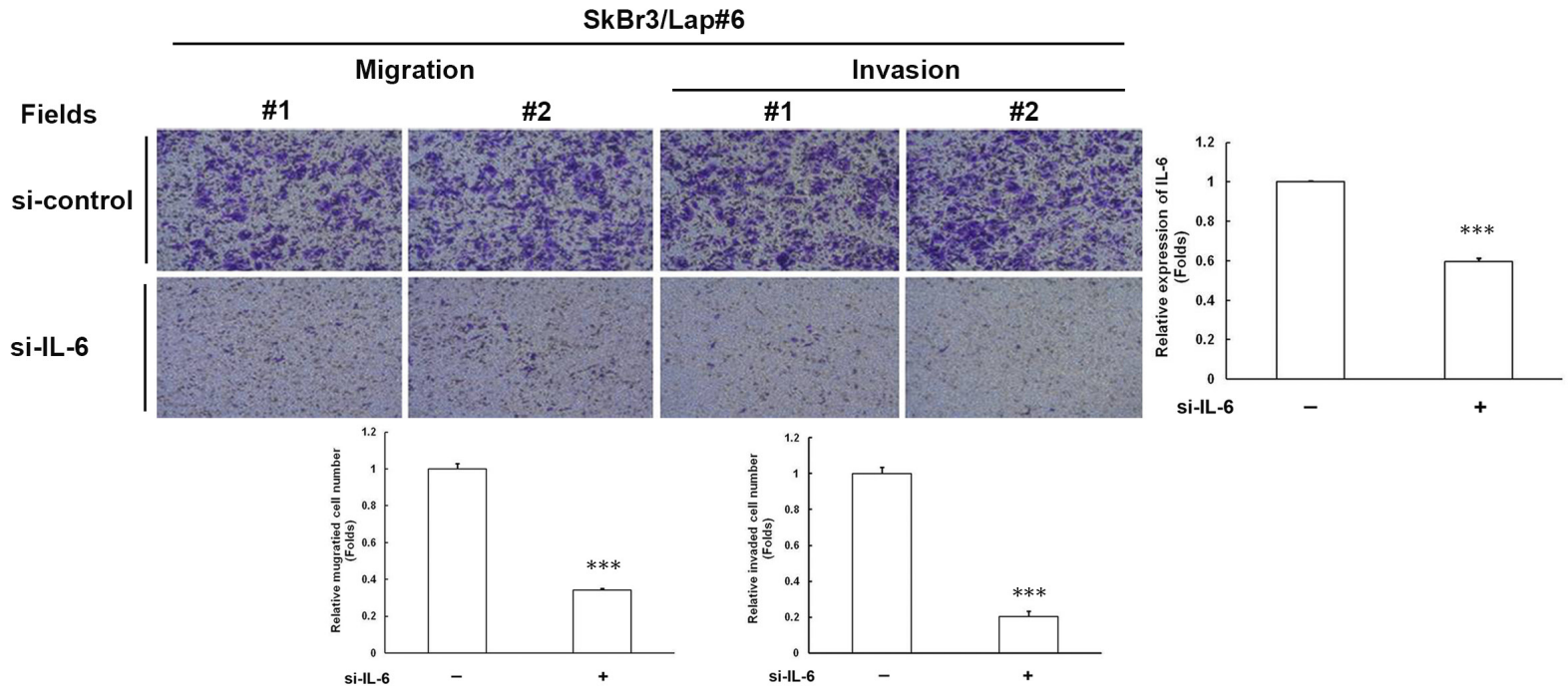

$\mathbf{F}$

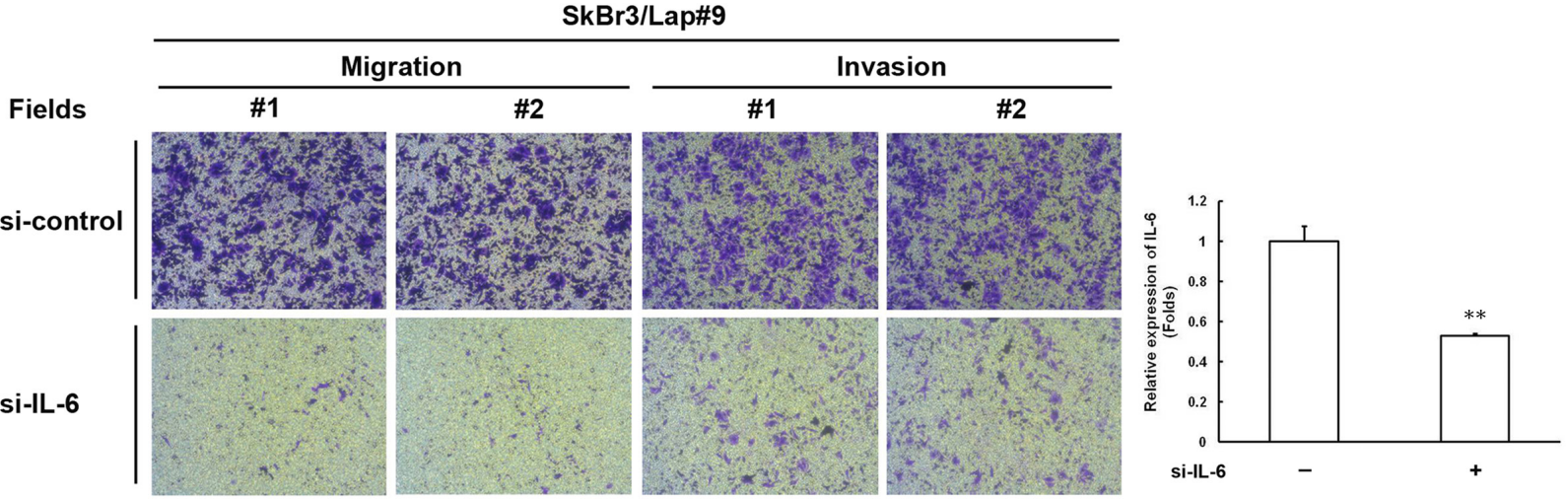

G

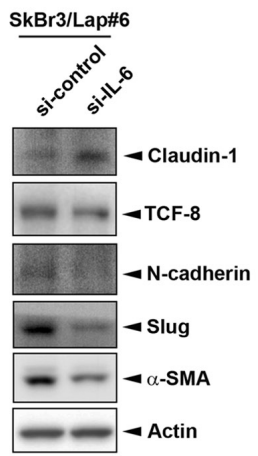

Figure 2 (Continued): E-F. SkBr3/Lap\#6 and SkBr3/Lap\#9 cells were transfected with si-control or si-IL6. Two days later, cells were re-seeded and subjected to in vitro transwell migration or invasion assay for $48 \mathrm{hrs}$. Representative pictures of migrated or invaded cells were visualized by crystal violet staining and quantified. Gene silencing of IL 6 was confirmed by ELISA. Statistical analysis was performed by Student's t test. **, $\mathrm{p}<0.01 ; * * *, \mathrm{p}<0.001$ as compared to control group. G. SkBr3/Lap\#6 cells were transfected with si-control or si-IL6. Four days later, whole cell lysates were harvested and subjected to western blot analysis with indicated antibodies. 
A

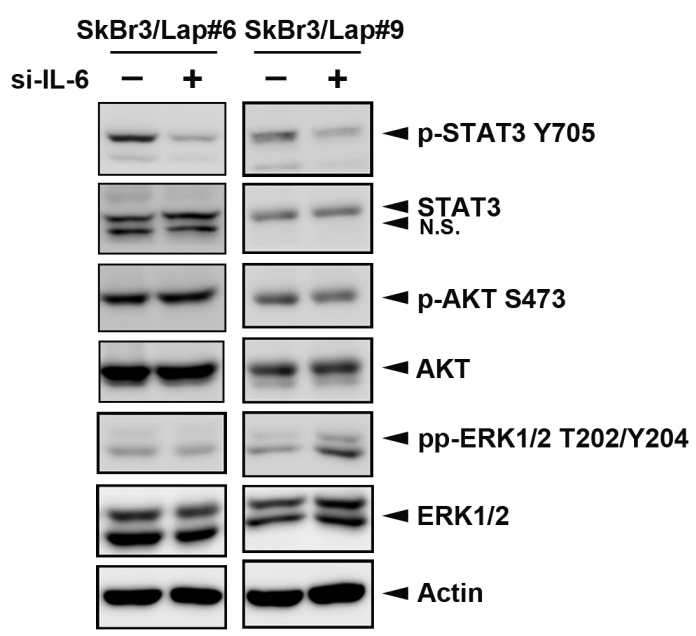

B

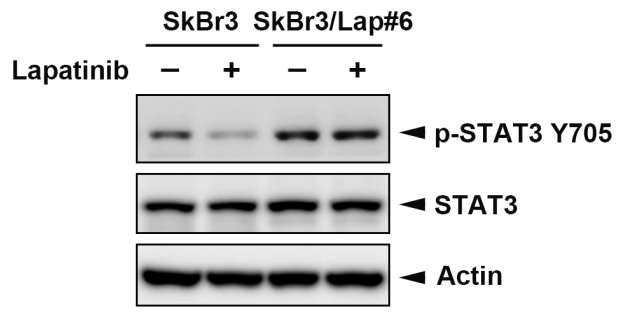

C
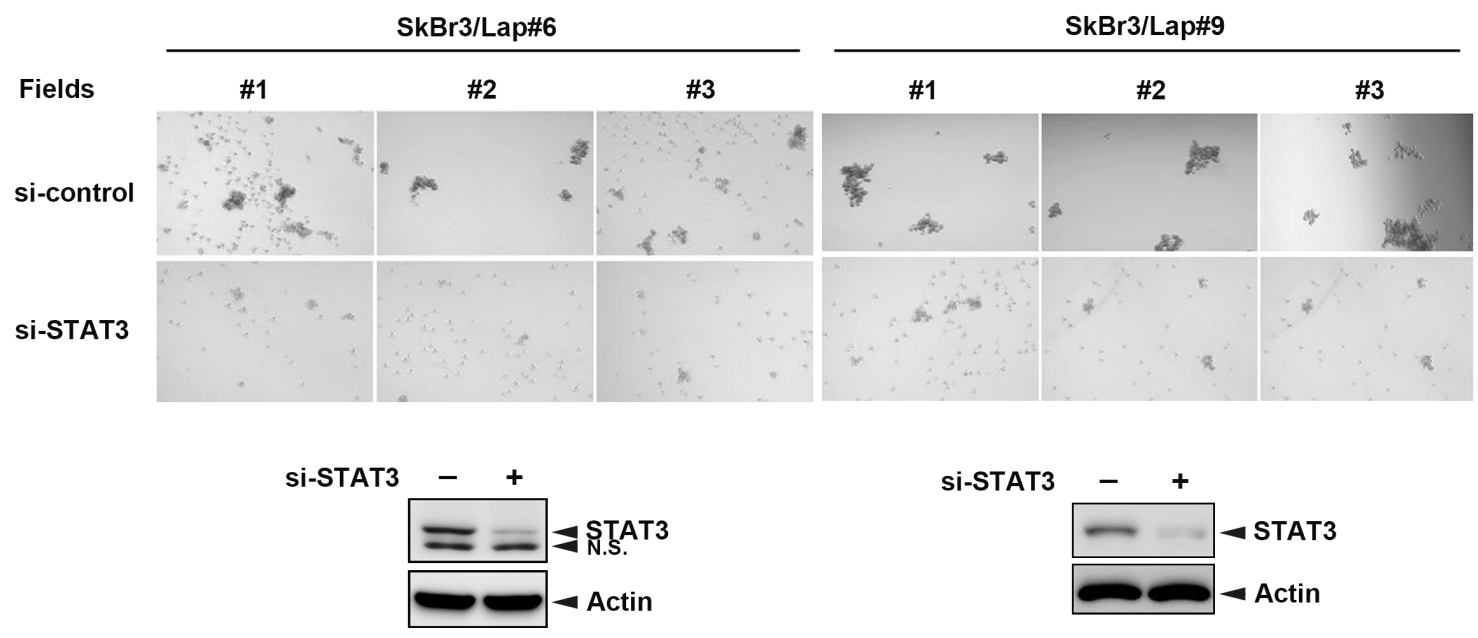

D
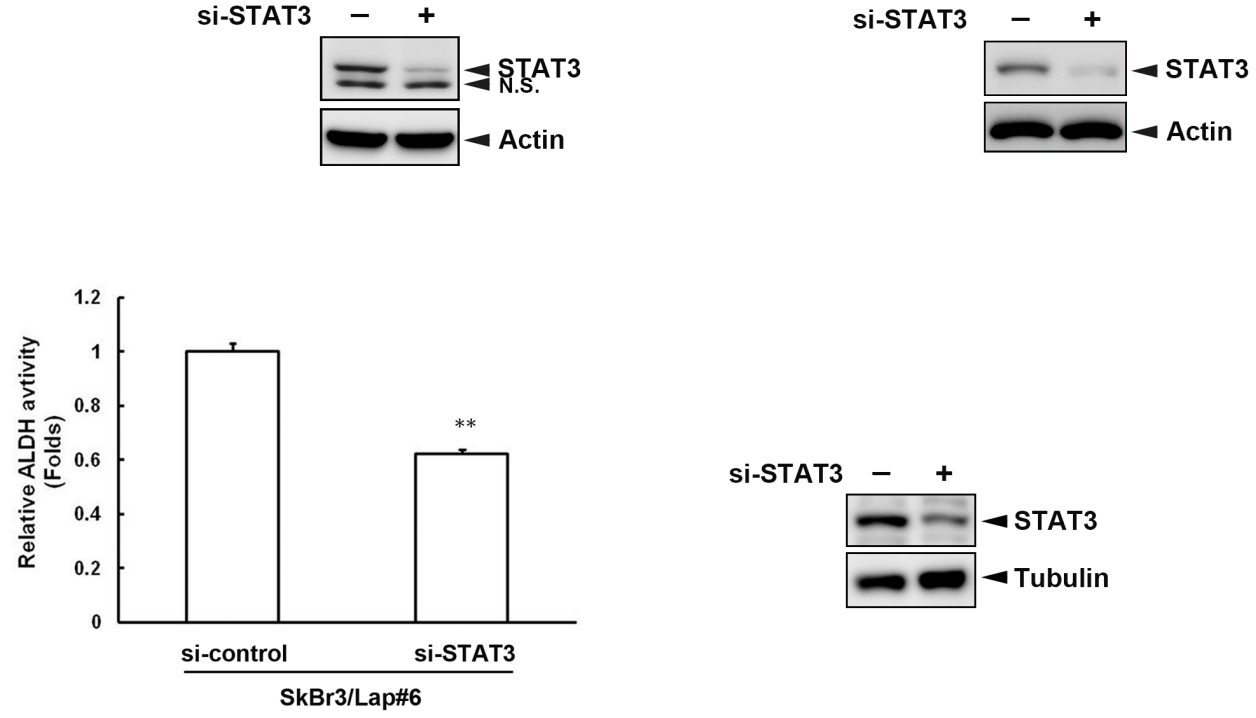

Figure 3: STAT3 activation is the major downstream effector for IL-6-mediated maintenance of stemness. A. SkBr3/ $\mathrm{Lap \# 6}$ and $\mathrm{SkBr3} / \mathrm{Lap} \# 9$ cells were transfected with si-control or si-IL6. Four days later, cells lysates were harvested and the expressions of several IL-6 downstream effectors were examined by western blot with indicated antibodies. B. SkBr3 and SkBr3/Lap\#6 cells were treated with $1 \mu \mathrm{M}$ lapatinib for $24 \mathrm{hrs}$. Cell lysates were collected and the expressions of phosphorylated STAT3 tyrosine 705 and STAT3 were examined by western blot. C. SkBr3/Lap\#6 and $\mathrm{SkBr3} / \mathrm{Lap} \# 9$ cells were transfected with si-control or si-STAT3. Two days later, cells were trypsinized and $4 \times 10^{4}$ cells were cultured in ultra-low attachment plates with spheroid-inducing medium, followed by the observation of spheroid formation under microscope. Gene silencing of STAT3 was examined by western blot. D. SkBr3/Lap\#6 cells were transfected with si-control or si-STAT3. Two days later, cells were trypsinized and $4 \times 10^{4}$ cells were cultured in ultra-low attachment plates with spheroidinducing medium for 7 days. Then, $2 \times 10^{6}$ spheroids were collected and subjected to an ALDH activity assay. Protein expression of STAT3 was examined by western blot. Statistical analysis was performed by Student's $t$ test. **, $p<0.01$ as compared to control group. 
decreased in both $\mathrm{SkBr3} / \mathrm{Lap} \# 6$ and $\mathrm{SkBr3} / \mathrm{Lap} \# 9$ cells (upper panel). Moreover, silencing of STAT3 expression reduced ALDH activity in SkBr3/Lap\#6 cells (Figure 3D). These results suggest that STAT3 activation is the major downstream effector for IL-6-mediated maintenance of stemness in acquired lapatinib-resistant cells.

\section{IL-6 activity is critical for lapatinib resistance}

To examine whether IL-6 is important for the survival of lapatinib-resistant cells, we inhibited IL-6 activity using both siRNA and neutralizing antibody. We found that the cell viability of lapatinib-resistant cells was decreased (Figure 4A, left panel) when IL6 expression was blocked by siRNA (Figure 4A, right panel). Similar results were also observed when IL-6 activity was inhibited by antibody (Figure 4B). We also increased IL-6 activity in parental SkBr3 cells with the addition of recombinant IL- 6 protein. The dosedependent cytotoxicity by lapatinib in $\mathrm{SkBr} 3$ cells without recombinant IL-6 was gradually reversed when cells were treated with higher doses of recombinant IL-6 protein (Figure 4C). In contrast, SkBr3/Lap\#6 cells became more sensitive to lapatinib treatment when IL-6 activity was inhibited by IL-6 antibody (Figure 4D). These results indicate that blocking IL-6 activity in resistant cells overcomes lapatinib resistance whereas stimulation of IL-6 activity in parental cells increases lapatinib resistance.

\section{DISCUSSION}

Targeted therapies, such as HER2-targeted therapy in breast cancer, are designed to directly attack the oncogenic proteins expressed by cancer cells and have been successful anti-cancer therapies [27]. Two types of HER2-targeted therapy, lapatinib and trastuzumab $\left(\right.$ Herceptin $\left.{ }^{\circledR}\right)$, are extensively used in clinic and show promising benefits for most HER2-positive breast cancer patients $[10,11]$. However, patients eventually develop acquired resistance, even after initially responding well to these drugs. This is a serious problem challenging anti-HER2 therapy in the clinic, and the mechanisms underlying resistance to anti-HER2 therapy have been intensively investigated [11-13, 28]. In this study, we found that long-term treatment of lapatinib in HER2-positive breast cancer cells led to increased IL6 expression, subsequent activation of downstream STAT3 signaling, and the maintenance of stemness, all of which contributed to acquired lapatinib resistance (Figures 1 \& 3). Targeting IL-6 activity reduced the BTIC population and subsequent cell viability of these resistant cells. In contrast, stimulation of IL-6 rendered parental cells more resistant to lapatinib treatment (Figures $2 \& 4$ ). Thus, a switch of survival signaling from HER 2 to IL-6 confers lapatinib resistance.
Mechanisms underlying resistance to lapatinib and trastuzumab are not always the same. Lapatinib resistance is mostly due to the switch of survival signaling from HER2 to other signals, such as AXL RTK [29], Src family kinase [30], etc. Moreover, the mechanism underlying lapatinib resistance may be different in HER2-positive breast cancer cells with or without estrogen receptor (ER) $[31,32]$. Our study identified a new alternative survival signaling pathway, IL-6, for lapatinib resistance. However, we explored the mechanism of lapatinib resistance in HER2-positive breast cancer cells without ER expression. Whether this resistance mechanism is also observed in ERpositive breast cancer cells deserves further investigation. For trastuzumab resistance, the underlying mechanisms usually arise from the inability of trastuzumab to bind to HER2. Since trastuzumab is only able to bind to HER2 homodimers, HER2 forming heterodimers with other up-regulated RTKs, such as insulin-like growth factor 1 receptor [33, 34], MET [35] and HER3 [36] may confer trastuzumab resistance. This may explain why breast cancer patients are still sensitive to lapatinib treatment after acquiring trastuzumab resistance [13]. Therefore, lapatinib may sometimes be a successful treatment for trastuzumab resistance [37].

Interestingly, Korkaya et al., also demonstrated that constitutive activation of the IL-6 signaling loop contributes to trastuzumab resistance through enlargement of the BTIC population [38]. It seems that IL-6 activity may be a general cause of resistance to anti-HER2 therapy. Simultaneous targeting of IL-6 and HER2 may be a strategy not only to prevent, but also to overcome, the resistance to anti-HER2 therapy. Our previous study showed that long-term treatment of lapatinib in breast cancer cells with or without HER2 expression enhances NF- $\mathrm{kB}$ activation and subsequently results in the expression of NF-KB downstream genes, including IL6 [26]. Furthermore, another more recent study by our group indicated that long-term treatment of lapatinib in triple-negative breast cancer cells increases IL6 expression through miRNA-7-dependent activation of Raf-1 signaling [39]. How IL6 expression is elevated in response to lapatinib resistance in this model awaits further clarification. On the other hand, whether other cytokine in addition to IL-6 is involved in lapatinib resistance deserves investigation. Building on these previous studies, this study not only explores a new mechanism underlying lapatinib resistance, but also provides a potential strategy to overcome this resistance via IL-6 inhibition.

\section{MATERIALS AND METHODS}

\section{Cell lines and reagents}

$\mathrm{SkBr} 3$ cells were maintained in Dulbecco's modified Eagle's medium/F12 medium supplemented with $10 \%$ fetal bovine serum (Logan, UT). Acquired 
A
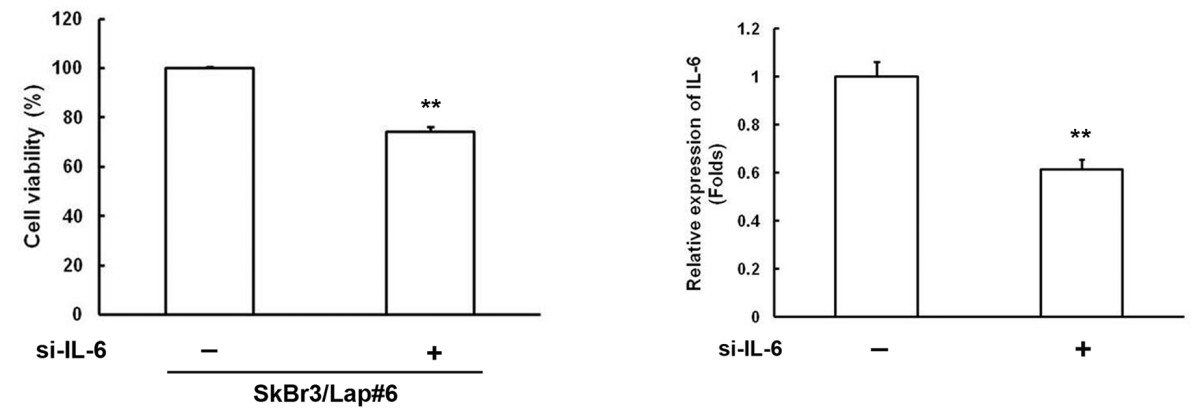

B

SkBr3/Lap\#6
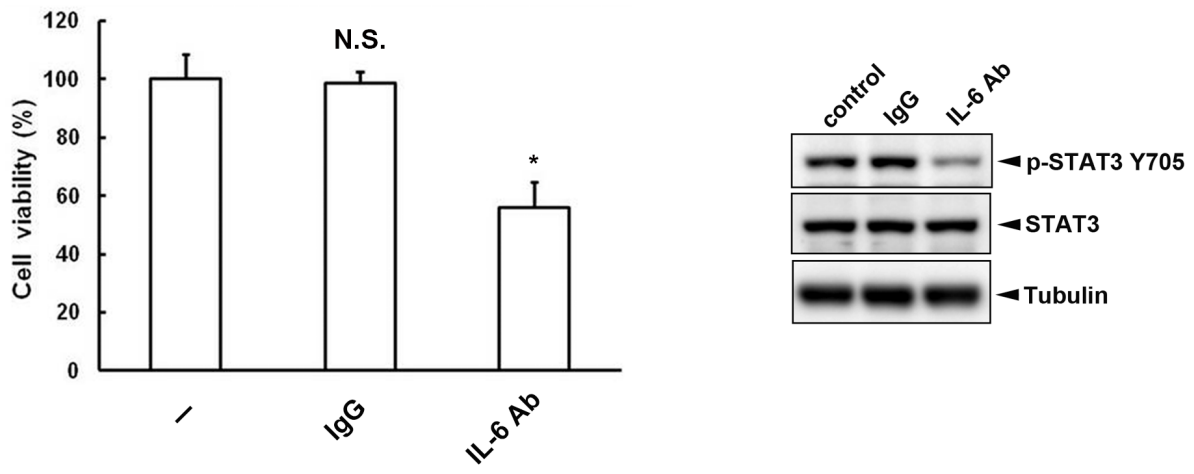

C

$\mathrm{SkBr3}$

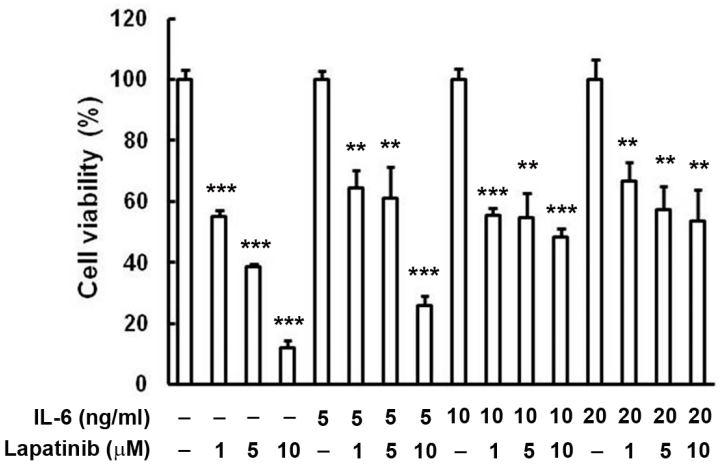

SkBr3/Lap\#6

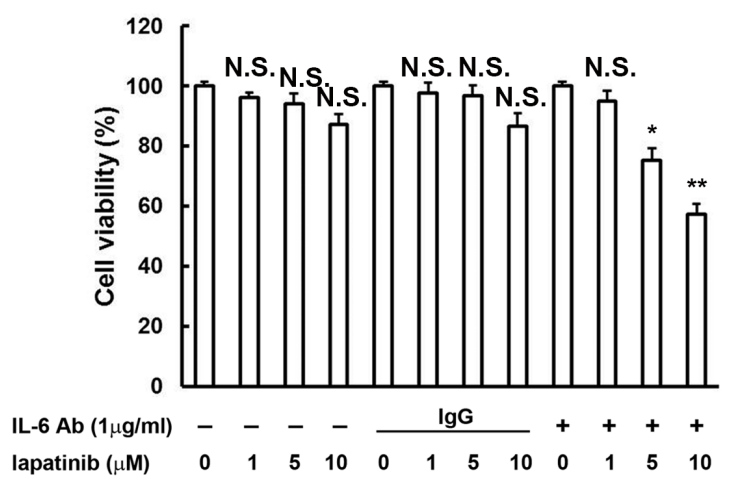

Figure 4: IL-6 activity is critical for lapatinib resistance. A. SkBr3/Lap\#6 cells were transfected with si-control or si-IL6. Two days later, cells were re-seeded at a density of $2 \times 10^{4}$ in a $6-\mathrm{cm}$ dish for 2 weeks. Cell viability was detected and quantified by crystal violet staining. Gene silencing of IL6 was confirmed by ELISA. Statistical analysis was performed by Student's t test. **, $\mathrm{p}<0.01$ as compared to control group. B. SkBr3/Lap\#6 cells were cultured at a density of 1 x $10^{4}$ cells in 96-well plates with medium treated with either $10 \mu \mathrm{g} /$ $\mathrm{ml} \mathrm{IgG} \mathrm{or} \mathrm{IL-6} \mathrm{antibody} \mathrm{for} 4$ days. Cell viability was examined by MTT assay. Cell lysates treated with $10 \mu \mathrm{g} / \mathrm{ml}$ IL-6 antibody were also collected to examine the expression of phosphorylated STAT3 tyrosine 705 by western blot. Statistical analysis was performed by Student's t test. *, p $<0.05$ as compared to control group. N.S. represents non-significant. C. SkBr3 cells were pre-treated with different concentrations of recombinant IL-6 protein, followed by treatment with the indicated concentrations of lapatinib for 3 days. Cell viability was then examined by MTT assay. Statistical analysis was performed by Student's t test. **, $\mathrm{p}<0.01 ; * * *, \mathrm{p}<0.001$ as compared to control group. D. SkBr3/Lap\#6 cells were pre-treated with or without IL-6 antibody, followed by treatment with the indicated concentrations of lapatinib for 3 days. Cell viability was then examined by MTT assay. Statistical analysis was performed by Student's t test. *, p $<0.05$; **, p $<0.01$ as compared to each control group. N.S. represents non-significant. 
lapatinib-resistant $\mathrm{SkBr} 3 / \mathrm{Lap} \# 6$ and $\mathrm{SkBr} 3 / \mathrm{Lap} \# 9$ cells were maintained in Dulbecco's modified Eagle's medium/F12 medium supplemented with $10 \%$ fetal bovine serum and $1 \mu \mathrm{M}$ lapatinib. The QuickGene RNA cultured cell kit was from Kurabo (Osaka, JP). The RevertAid ${ }^{\mathrm{TM}}$ H Minus First Strand cDNA synthesis kit was purchased from Thermo Fisher Scientific (Waltham, MA). The ALDH activity colorimetric assay kit was from BioVision (Milpitas, CA). Si-control, si-IL6 and si-STAT3 as well as antibodies against Tubulin and Actin were purchased from Sigma-Aldrich (St. Louis, MO). TransIT-2020 transfection reagent from Mirus Bio LLC (Madison, WI) was used. Transwell chambers (24-well insert; pore size, $8 \mathrm{~mm}$ ) were purchased from Costar Corp. (Cambridge, MA). Human sIL-6 instant ELISA kit was from eBioscience (San Diego, CA). The ultra-low attachment multi-well plates were purchased from Corning (New York). The IL-6 recombinant protein was from Miltenyi Biotec (Bergisch Gladbach, Germany). The matrigel basement membrane matrix from BD Biosciences (East Rutherford, NJ) was used. The antibodies against phospho-Akt serine 473, Akt, phospho-STAT3 tyrosine 705 and phospho-ERK1/2 threonine 202/tyrosine 204 were from Cell Signaling (Danvers, MA). The antibody against STAT3 was purchased from Santa Cruz Biotechnology Inc. (Santa Cruz, CA). The antibody against ERK1/2 from Millipore (Darmstadt, Germany) was used. The IL-6 antibody was purchased from R\&D Systems (Minneapolis, MN).

\section{Reverse transcription-polymerase chain reaction (RT-PCR)}

The QuickGene RNA cultured cell kit was used for total RNA extraction according to manufacturer's instructions as described previously [40]. Briefly, $1 \mu \mathrm{g}$ of RNA was used for reverse transcription using the RevertAid $^{\mathrm{TM}} \mathrm{H}$ Minus First Strand cDNA synthesis kit. PCR analysis of IL6 mRNA expression was performed and visualized by $1 \%$ gel electrophoresis. ACTIN mRNA expression was used as an internal control. The primer sequences of IL-6 are as follows: forward 5'ATGAACTCCTTCTCCACAAGCGC -3'; reverse 5'GAAGAGCCCTCAGGATGGACTG -3'. The primer sequences of ACTIN are as follows: forward 5'GGGTCAGAAGGATTCCTATG -3'; reverse 5'- GGTC TCAAACATGATCTGGG -3'.

\section{Enzyme linked immunosorbent assay (ELISA)}

The production of IL-6 was detected by using human sIL-6 instant ELISA kit according to manufacturer's instructions. In brief, the number of microwell strips for the experiment was determined and the pellets were dissolved thoroughly by adding 100 $\mu \mathrm{l}$ of distilled water. Then, $50 \mu \mathrm{l}$ samples, in duplicate, were added to designated wells and mixed with the contents. The microwell strips were covered by adhesive film and incubated at room temperature. After 3-hour incubation, microwell strips were mixed and washed thoroughly with $400 \mu \mathrm{l}$ wash buffer (6 times). After the final wash, the excess buffer in the microwell strips was completely removed and $100 \mu \mathrm{TMB}$ substrate solution was added to each well, followed by incubation at room temperature for 1-10 minutes. The $100 \mu 1$ stop solution was not added until the complete reaction was finished and the value of absorbance was determined immediately at $450 \mathrm{~nm}$.

\section{Spheroid formation assay}

Cells were trypsinized and washed with $1 \mathrm{X}$ PBS twice and with spheroid-inducing medium once. Then, cells at a density of $2 \times 10^{4}$ cells $/ \mathrm{ml}$ were seeded in an ultra-low attachment plate with spheroid-inducing medium. Cells were filtered and given new spheroidinducing medium every week, allowing the formation of spheroids. The number of spheroids were then determined under microscope.

\section{Aldehyde dehydrogenase (ALDH) activity colorimetric assay}

The ALDH activity was determined using ALDH activity colorimetric assay kit according to the manufacturer's instructions. Briefly, 2 x $10^{6}$ cells were rapidly homogenized with $200 \mu$ of ice cold ALDH assay buffer for 10 minutes on ice. Then, cells were spun down at 12,000 rpm for 5 minutes to remove nuclei and insoluble material. $50 \mu \mathrm{l}$ of collected supernatants was loaded into a 96-well plate and the final volume was adjusted to 50 $\mu \mathrm{l}$ with ALDH assay buffer. Then, $50 \mu \mathrm{l}$ of reaction mix containing ALDH assay buffer, ALDH substrate mix, and acetaldehyde was added to each well. The mixture was incubated at room temperature for 5 minutes, followed by the measurement of absorbance at $450 \mathrm{~nm}$.

\section{Transfection of siRNA}

Cells at $60-80 \%$ of confluence were transfected with siRNA in a final concentration of $100 \mathrm{nM}$ by using TransIT-2020 transfection reagent according to the manufacturer's instructions as described previously [41]. After 2-days, cells were subjected to further experiments, including the spheroid formation assay, the ALDH activity assay, in vitro transwell assay, and the cell viability assay. Cell lysates and ELISA assay for confirmation of gene silencing were harvested and performed 4 days after transfections.

\section{In vitro transwell migration and invasion assays}

Cell migration and invasion were determined by transwell assay as described previously [40]. For the 
migration assay, cells at a density of $5 \times 10^{4} /$ well were seeded on the non-coated membrane of the upper chamber. For invasion assay, the membrane of the upper chamber was coated with matrigel for $1 \mathrm{hr}$ before $5 \times 10^{4}$ cells per well were seeded on the matrigel-coated membrane. After 48-hr incubation, cells were washed with 1X PBS once, followed by fixation with $4 \%$ formaldehyde. Thirty minutes later, cells were washed with $1 \mathrm{X}$ PBS once again and stained with $1 \%$ crystal violet in a solvent of $30 \%$ ethanol for 15-30 minutes at room temperature. Cells remaining on the upper chamber were removed using a cotton swab. The number of cells migrating or invading through the pores to the opposite side of the membrane was quantified.

\section{Cell viability assays}

Cell viability assay was assessed using both the clonogenic and the 3-(4,5-dimethylthiazol-2-yl)-2,5diphenyltetrazolium bromide (MTT) colorimetric assays. For the clonogenic assay, cells were re-seeded at a density of $2 \times 10^{4} / 6-\mathrm{cm}$ dish and allowed to grow for 2 weeks. Cell viability was detected and quantified by crystal violet staining. For MTT assay, 5 x $10^{3}$ cells/well were seeded on 96-well plates overnight and then pre-treated with indicated drugs. Three to four days later, relative cell amounts were determined by adding $1 \mu \mathrm{g} / \mathrm{ml}$ MTT to each well. Then, the medium was removed after 4-hour incubation and formazan was solubilized in $100 \mu \mathrm{DMSO}$ per well, followed by the measurement of absorbance at $570 \mathrm{~nm}$.

\section{Statistical analysis}

The statistical analysis was performed by Student's $t$ test. $*, p<0.05$; **, $p<0.01$; ***, $p<0.001$ mean as compared to each control groups.

\section{CONFLICTS OF INTEREST}

The authors declare no conflicts of interest regarding the publication of this article.

\section{FINANCIAL SUPPORT}

This work was supported by grants from the Ministry of Science and Technology, R.O.C. (MOST-1052320-B-039-056-MY3, MOST-103-2320-B-214-005, MOST-103-2320-B-039-001 and MOST-1052320-B-214-005), I-Shou University (ISU-103-04-07A), E-Da Hospital (EDPJ104070 and EDAHP104060), China Medical University (CMU-104-S-01) and the National Health Research Institutes of Taiwan (NHRIEX104-10329BI).

\section{REFERENCES}

1. Vargo-Gogola T, Rosen JM Modelling breast cancer: one size does not fit all. Nat Rev Cancer 2007; 7: 659-672.

2. Eroles P, Bosch A, Perez-Fidalgo JA, Lluch A Molecular biology in breast cancer: intrinsic subtypes and signaling pathways. Cancer Treat Rev 2012; 38: 698-707.

3. Hynes NE, Lane HA ERBB receptors and cancer: the complexity of targeted inhibitors. Nat Rev Cancer 2005; 5 : 341-354.

4. Paik S, Kim C, Wolmark N HER2 status and benefit from adjuvant trastuzumab in breast cancer. N Engl J Med 2008; 358: 1409-1411.

5. Li X, Lewis MT, Huang J, Gutierrez C, Osborne CK, Wu MF, Hilsenbeck SG, Pavlick A, Zhang X, Chamness GC, Wong $\mathrm{H}$, Rosen $\mathrm{J}$, et al. Intrinsic resistance of tumorigenic breast cancer cells to chemotherapy. J Natl Cancer Inst 2008; 100: 672-679.

6. Korkaya H, Paulson A, Iovino F, Wicha MS HER2 regulates the mammary stem/progenitor cell population driving tumorigenesis and invasion. Oncogene 2008; 27: 6120-6130.

7. Ginestier C, Hur MH, Charafe-Jauffret E, Monville F, Dutcher J, Brown M, Jacquemier J, Viens P, Kleer CG, Liu $\mathrm{S}$, Schott A, Hayes D, et al. ALDH1 is a marker of normal and malignant human mammary stem cells and a predictor of poor clinical outcome. Cell Stem Cell 2007; 1: 555-567.

8. Freudenberg JA, Wang Q, Katsumata M, Drebin J, Nagatomo I, Greene MI The role of HER2 in early breast cancer metastasis and the origins of resistance to HER2targeted therapies. Exp Mol Pathol 2009; 87: 1-11.

9. Eccles SA The epidermal growth factor receptor/Erb-B/ HER family in normal and malignant breast biology. Int $\mathrm{J}$ Dev Biol 2011; 55: 685-696.

10. Awada A, Bozovic-Spasojevic I, Chow L New therapies in HER2-positive breast cancer: a major step towards a cure of the disease? Cancer Treat Rev 2012; 38: 494-504.

11. Nielsen DL, Andersson M, Kamby C HER2-targeted therapy in breast cancer. Monoclonal antibodies and tyrosine kinase inhibitors. Cancer Treat Rev 2009; 35: 121-136.

12. Rexer BN, Arteaga CL Intrinsic and acquired resistance to HER2-targeted therapies in HER2 gene-amplified breast cancer: mechanisms and clinical implications. Crit Rev Oncog 2012; 17: 1-16.

13. Garrett JT, Arteaga CL Resistance to HER2-directed antibodies and tyrosine kinase inhibitors: mechanisms and clinical implications. Cancer Biol Ther 2011; 11: 793-800.

14. Muraguchi A, Kishimoto T, Miki Y, Kuritani T, Kaieda T, Yoshizaki K, Yamamura Y T cell-replacing factor- (TRF) induced $\operatorname{IgG}$ secretion in a human B blastoid cell line and demonstration of acceptors for TRF. J Immunol 1981; 127: 412-416. 
15. Yoshizaki K, Nakagawa T, Kaieda T, Muraguchi A, Yamamura $\mathrm{Y}$, Kishimoto $\mathrm{T}$ Induction of proliferation and Ig production in human B leukemic cells by antiimmunoglobulins and T cell factors. J Immunol 1982; 128: 1296-1301.

16. Guo Y, Xu F, Lu T, Duan Z, Zhang Z Interleukin-6 signaling pathway in targeted therapy for cancer. Cancer Treat Rev 2012; 38: 904-910.

17. Barton BE Interleukin-6 and new strategies for the treatment of cancer, hyperproliferative diseases and paraneoplastic syndromes. Expert Opin Ther Targets 2005; 9: 737-752.

18. Knupfer H, Preiss R Significance of interleukin-6 (IL-6) in breast cancer (review). Breast Cancer Res Treat 2007; 102: 129-135.

19. Suh YA, Jo SY, Lee HY, Lee C Inhibition of IL-6/STAT3 axis and targeting Axl and Tyro3 receptor tyrosine kinases by apigenin circumvent taxol resistance in ovarian cancer cells. Int J Oncol 2015; 46: 1405-1411.

20. Yang L, Han S, Sun Y An IL6-STAT3 loop mediates resistance to $\mathrm{PI} 3 \mathrm{~K}$ inhibitors by inducing epithelialmesenchymal transition and cancer stem cell expansion in human breast cancer cells. Biochem Biophys Res Commun 2014; 453: 582-587.

21. Yan HQ, Huang XB, Ke SZ, Jiang YN, Zhang YH, Wang YN, Li J, Gao FG Interleukin 6 augments lung cancer chemotherapeutic resistance via ataxia-telangiectasia mutated/NF-kappaB pathway activation. Cancer Sci 2014; 105: 1220-1227.

22. Hartman ZC, Yang XY, Glass O, Lei G, Osada T, Dave SS, Morse MA, Clay TM, Lyerly HK HER2 overexpression elicits a proinflammatory IL-6 autocrine signaling loop that is critical for tumorigenesis. Cancer Res 2011; 71: 4380-4391.

23. Zhao S, Ohara S, Kanno Y, Midorikawa Y, Nakayama M, Makimura M, Park Y, Inouye Y HER2 overexpressionmediated inflammatory signaling enhances mammosphere formation through up-regulation of aryl hydrocarbon receptor transcription. Cancer Lett 2013; 330: 41-48.

24. Sansone P, Storci G, Tavolari S, Guarnieri T, Giovannini C, Taffurelli M, Ceccarelli C, Santini D, Paterini P, Marcu $\mathrm{KB}$, Chieco P, Bonafe M IL-6 triggers malignant features in mammospheres from human ductal breast carcinoma and normal mammary gland. J Clin Invest 2007; 117: 3988-4002.

25. Goldberg JE, Schwertfeger KL Proinflammatory cytokines in breast cancer: mechanisms of action and potential targets for therapeutics. Curr Drug Targets 2010; 11: 1133-1146.

26. Chen YJ, Yeh MH, Yu MC, Wei YL, Chen WS, Chen JY, Shih CY, Tu CY, Chen CH, Hsia TC, Chien PH, Liu SH, et al. Lapatinib-induced NF-kappaB activation sensitizes triple-negative breast cancer cells to proteasome inhibitors. Breast Cancer Res 2013; 15: R108.
27. Tagliabue E, Balsari A, Campiglio M, Pupa SM HER2 as a target for breast cancer therapy. Expert Opin Biol Ther 2010; 10: 711-724.

28. Nahta R, Yu D, Hung MC, Hortobagyi GN, Esteva FJ Mechanisms of disease: understanding resistance to HER2targeted therapy in human breast cancer. Nat Clin Pract Oncol 2006; 3: 269-280.

29. Liu L, Greger J, Shi H, Liu Y, Greshock J, Annan R, Halsey W, Sathe GM, Martin AM, Gilmer TM Novel mechanism of lapatinib resistance in HER2-positive breast tumor cells: activation of AXL. Cancer Res 2009; 69: 6871-6878.

30. Rexer BN, Ham AJ, Rinehart C, Hill S, Granja-Ingram Nde M, Gonzalez-Angulo AM, Mills GB, Dave B, Chang JC, Liebler DC, Arteaga CL Phosphoproteomic mass spectrometry profiling links Src family kinases to escape from HER2 tyrosine kinase inhibition. Oncogene 2011; 30: 4163-4174.

31. Xia W, Bacus S, Hegde P, Husain I, Strum J, Liu L, Paulazzo G, Lyass L, Trusk P, Hill J, Harris J, Spector NL A model of acquired autoresistance to a potent ErbB2 tyrosine kinase inhibitor and a therapeutic strategy to prevent its onset in breast cancer. Proc Natl Acad Sci U S A 2006; 103 : 7795-7800.

32. Xia W, Bacus S, Husain I, Liu L, Zhao S, Liu Z, Moseley MA, 3rd, Thompson JW, Chen FL, Koch KM, Spector NL Resistance to ErbB2 tyrosine kinase inhibitors in breast cancer is mediated by calcium-dependent activation of RelA. Mol Cancer Ther 2010; 9: 292-299.

33. Lu Y, Zi X, Zhao Y, Mascarenhas D, Pollak M Insulinlike growth factor-I receptor signaling and resistance to trastuzumab (Herceptin). J Natl Cancer Inst 2001; 93: 1852-1857.

34. Nahta R, Yuan LX, Zhang B, Kobayashi R, Esteva FJ Insulin-like growth factor-I receptor/human epidermal growth factor receptor 2 heterodimerization contributes to trastuzumab resistance of breast cancer cells. Cancer Res 2005; 65: 11118-11128.

35. Shattuck DL, Miller JK, Carraway KL, 3rd, Sweeney C Met receptor contributes to trastuzumab resistance of Her2overexpressing breast cancer cells. Cancer Res 2008; 68: 1471-1477.

36. Wang SE, Xiang B, Guix M, Olivares MG, Parker J, Chung $\mathrm{CH}$, Pandiella A, Arteaga CL Transforming growth factor beta engages TACE and ErbB3 to activate phosphatidylinositol-3 kinase/Akt in ErbB2-overexpressing breast cancer and desensitizes cells to trastuzumab. Mol Cell Biol 2008; 28: 5605-5620.

37. Geyer CE, Forster J, Lindquist D, Chan S, Romieu CG, Pienkowski T, Jagiello-Gruszfeld A, Crown J, Chan A, Kaufman B, Skarlos D, Campone M, et al. Lapatinib plus capecitabine for HER2-positive advanced breast cancer. N Engl J Med 2006; 355: 2733-2743.

38. Korkaya H, Kim GI, Davis A, Malik F, Henry NL, Ithimakin S, Quraishi AA, Tawakkol N, D'Angelo R, Paulson AK, Chung S, Luther T, et al. Activation of an IL6 inflammatory 
loop mediates trastuzumab resistance in HER2+ breast cancer by expanding the cancer stem cell population. Mol Cell 2012; 47: 570-584.

39. Hsiao YC, Yeh MH, Chen YJ, Liu JF, Tang CH, Huang WC Lapatinib increases motility of triple-negative breast cancer cells by decreasing miRNA-7 and inducing Raf-1/MAPKdependent interleukin-6. Oncotarget 2015; 6: 37965-37978. doi: 10.18632/oncotarget.5700.

40. Hung CM, Huang WC, Pan HL, Chien PH, Lin CW, Chen LC, Chien YF, Lin CC, Leow KH, Chen WS, Chen JY, Ho $\mathrm{CY}$, et al. Hepatitis B virus X upregulates HuR protein level to stabilize HER2 expression in hepatocellular carcinoma cells. Biomed Res Int 2014; 2014: 827415.

41. Huang WC, Hsieh YL, Hung CM, Chien PH, Chien YF, Chen LC, Tu CY, Chen CH, Hsu SC, Lin YM, Chen YJ BCRP/ABCG2 inhibition sensitizes hepatocellular carcinoma cells to sorafenib. PLoS One 2013; 8: e83627. 